\title{
THE STRUCTURE OF THE LARGE MAGELLANIC CLOUD STELLAR HALO DERIVED USING OGLE-III RR Lyr STARS
}

\author{
Ondřej Pejcha And K. Z. StaneK \\ Department of Astronomy, The Ohio State University, 140 West 18th Avenue, Columbus, OH 43210, USA; pejcha@astronomy.ohio-state.edu, \\ kstanek@astronomy.ohio-state.edu \\ Received 2009 May 21; accepted 2009 September 14; published 2009 October 6
}

\begin{abstract}
We use the recently released OGLE-III catalog of 17,692 fundamental mode RR Lyr (RRab) stars in the Large Magellanic Cloud (LMC) to investigate the structure of its stellar halo. We apply conservative cuts in period, amplitude, and magnitude to remove blends and other contamination. We use period-luminosity and period-color relations to determine distance and extinction of every star in our final sample of 9393 stars. In order to determine the scatter of our method, we compare the distributions of distances in two regions at the edges of the covered area with a central region. We determine the intrinsic line-of-sight dispersion in the center to be $0.135 \mathrm{mag}$ or $3.21 \mathrm{kpc}$ (FWHM of $0.318 \mathrm{mag}$ or $7.56 \mathrm{kpc}$ ), assuming zero depth in one of the edge regions. The conservative cuts we apply reduce the derived depth significantly. Furthermore, we find that the distribution of RRab stars is deformed in the sense that stars on the eastern side are closer than on the western side. We model the RRab distribution as a triaxial ellipsoid and determine its axes ratios to be $1: 2.00: 3.50$ with the longest axis inclined by $6^{\circ}$ from the line of sight. Another result of our analysis is an extinction map of the LMC and a map of internal reddening, which we make publicly available.
\end{abstract}

Key words: galaxies: structure - Magellanic Clouds - stars: variables: other

\section{INTRODUCTION}

The structure and evolution of the Large Magellanic Cloud (LMC) stellar populations has been thoroughly investigated using many different methods (e.g., van der Marel et al. 2002; Nikolaev et al. 2004; Lah et al. 2005; Cole et al. 2005). One of the least studied populations remains the stellar halo, for which the preferred tracer objects are old metal-poor RR Lyr variable stars (Kinman et al. 1991). Minniti et al. (2003) and Borissova et al. $(2004,2006)$ measured radial velocity dispersions of a number of RR Lyr stars to be $\sim 50 \mathrm{~km} \mathrm{~s}^{-1}$, compared to e.g., $20 \mathrm{~km} \mathrm{~s}^{-1}$ for carbon stars (van der Marel et al. 2002). This suggests that RR Lyr stars form a kinematically hot halo with a significant vertical spread.

In this study, we use the OGLE-III catalog of the fundamental mode RR Lyr (RRab) stars (Soszyński et al. 2009) to investigate the structure of the stellar halo of the LMC. The advantage of RRab stars is that distances to individual stars can be estimated using the period-luminosity relation and the extinction can be determined using the period-color relation.

In Section 2, we describe the data set and how distance and extinction are estimated for individual stars. In Section 3, we present the extinction map and the three-dimensional structure of the LMC. In Section 4, we discuss the possible systematics of our method, compare our findings to previous results on the structure of the LMC and discuss the implications of our results.

\section{DATA AND METHOD}

To study the structure of the LMC old stellar population we use 9393 RRab stars. We start with 17,692 candidate RRab stars selected from the catalog of Soszyński et al. (2009), who also provided the pulsation mode classification based on Fourier coefficients of the light curves. We then exclude stars that were flagged as uncertain, foreground, or belonging to one of the LMC globular clusters. We applied further cuts to reduce the number of blended stars, likely misclassifications and stars with extreme properties. Specifically, we keep only stars with periods 0.45 days $\leqslant P \leqslant 0.70$ days, amplitudes $0.30 \mathrm{mag} \leqslant \Delta I \leqslant 0.85 \mathrm{mag}$ and $I \geqslant 18 \mathrm{mag}$. Furthermore, we remove the approximately $9 \%$ of stars that fall outside the main period-amplitude sequence shown in Figure 3 of Soszyński et al. (2009). The equatorial positions $(\alpha, \delta)$ of the remaining 11,171 stars are plotted in Figure 1. The edges of the OGLE-III coverage are rather irregular with respect to the RR Lyr distribution. To prevent appearance of artifacts in projected density plots we remove stars that fall outside a rectangle of size $8.4 \times 3.1$ and oriented parallel to the long axis of RRab distribution on the sky. This leaves 9393 stars as our final sample. The rectangle is shown in black in Figure 1 and the method for determining the shape of the RRab distribution is discussed in Section 3. The centroid position of our final sample is $\left(\alpha_{0}, \delta_{0}\right)=(80.35,69.68)$.

In order to estimate distances to individual RRab stars in the LMC we make use of the period-luminosity and periodcolor relations determined from RRab stars in the galactic globular cluster M3. We take advantage of the fact that the properties of RRab stars in this cluster are similar to the LMC, namely the mean period and the position of ridgelines in the period-amplitude diagram (Alcock et al. 2000a; Clement et al. 2008). Furthermore, the extinction toward M3 is very small, $E(B-V) \approx 0.01 \mathrm{mag}$ (Cacciari et al. 2005; Schlegel et al. 1998). For the slope of the period-luminosity relation $\eta$ we use the value determined by Benkô et al. (2006), namely $\eta=1.453$. They also found that the scatter of their period-luminosity relation is 0.04 mag. From the data set of Benkó et al. (2006) we determined the relation between period and intrinsic color $(V-I)_{0}$ using robust linear least-squares fit. The period-color relation is

$$
(V-I)_{0}=0.69+0.89 \log P
$$

with an rms scatter of 0.062 mag.

The $I$-band extinction $A_{I}$ to a particular star is estimated from the difference between the observed color index $(V-I)$ and the 


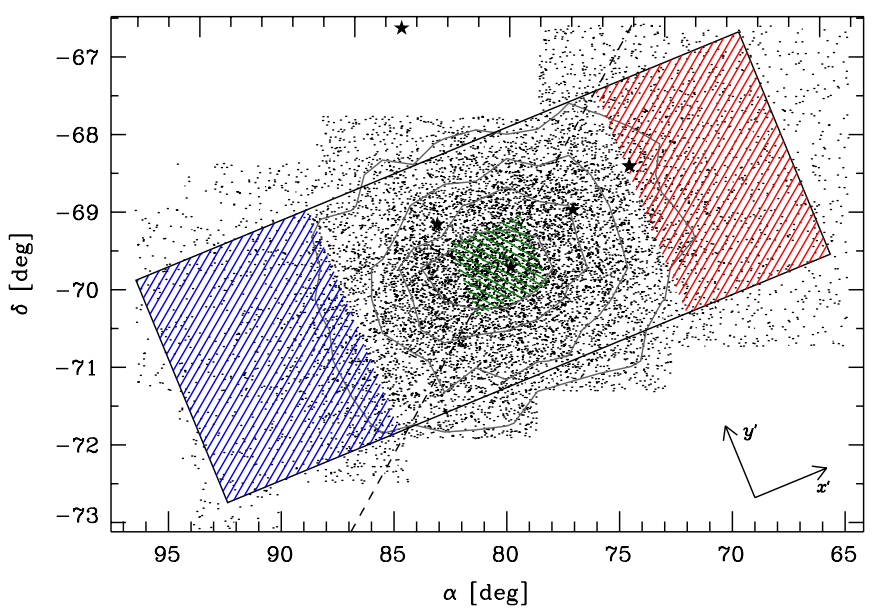

Figure 1. Positions of the 11,171 RRab stars satisfying cuts on period, amplitude and magnitude. Density contours marking levels of 250,500,750, and 1000 stars per square degree are shown with gray lines. The rectangle containing the 9393 stars of our final sample is shown in black. Regions that are investigated more closely are marked with blue, green and red parallel lines. The line of nodes measured by Nikolaev et al. (2004) is shown with a black dashed line going through the centroid position of our sample $\left(\alpha_{0}, \delta_{0}\right)$. Black filled stars show positions of eclipsing binaries with measured distances; in order of increasing $\alpha$ : HV 2274 (Ribas et al. 2000; Groenewegen \& Salaris 2001), OGLE-0510 (Pietrzyński et al. 2009), EROS 1044 (Ribas et al. 2002), HV 982 (Fitzpatrick et al. 2002) and HV 5936 (Fitzpatrick et al. 2003).

(A color version of this figure is available in the online journal.)

intrinsic color using

$$
A_{I}=R_{V I}\left[(V-I)-(V-I)_{0}\right],
$$

where $R_{V I}=R_{I} /\left(R_{V}-R_{I}\right)$ is the ratio between total $I$-band extinction and reddening of the $(V-I)$ color. If we get $A_{I}<0$ from Equation (2), we manually set $A_{I}=0$. Throughout this paper, we set $R_{V I}=1.10$, a slightly lower value than the usual $R_{V I}=1.55$ (e.g., Schlegel et al. 1998), to avoid inconsistencies in extinction estimates. We discuss implications of different values of $R_{V I}$ in Section 4.1.

Using the information provided we are able to correct the observed intensity-mean $I$ magnitude for extinction $A_{I}$ and for period-luminosity relation according to

$$
I_{0}^{\prime}=I-\eta \log P-A_{I} .
$$

The line-of-sight distance $z$ to a particular star is then obtained from $I_{0}^{\prime}$ assuming that the median distance of our final sample is $50 \mathrm{kpc}$. Our method resembles the approach of Nikolaev et al. (2004) who studied the LMC Cepheids.

\section{THE THREE-DIMENSIONAL STRUCTURE OF THE LMC}

In this section, we first create an extinction map of the LMC and estimate the internal reddening. Then we focus on the threedimensional structure of the stellar halo of the LMC as traced by RRab stars.

\subsection{Extinction Map and Internal Reddening}

We construct the extinction map ${ }^{1}$ of the LMC by dividing the plane of the sky into pixels of $0.2 \times 0.2$ and calculating mean $A_{I}$ for every pixel containing at least two stars. The extinction map

\footnotetext{
1 The extinction map can be obtained at http://www.astronomy.ohio-state.edu/ pejcha/lmc_extmap.
}
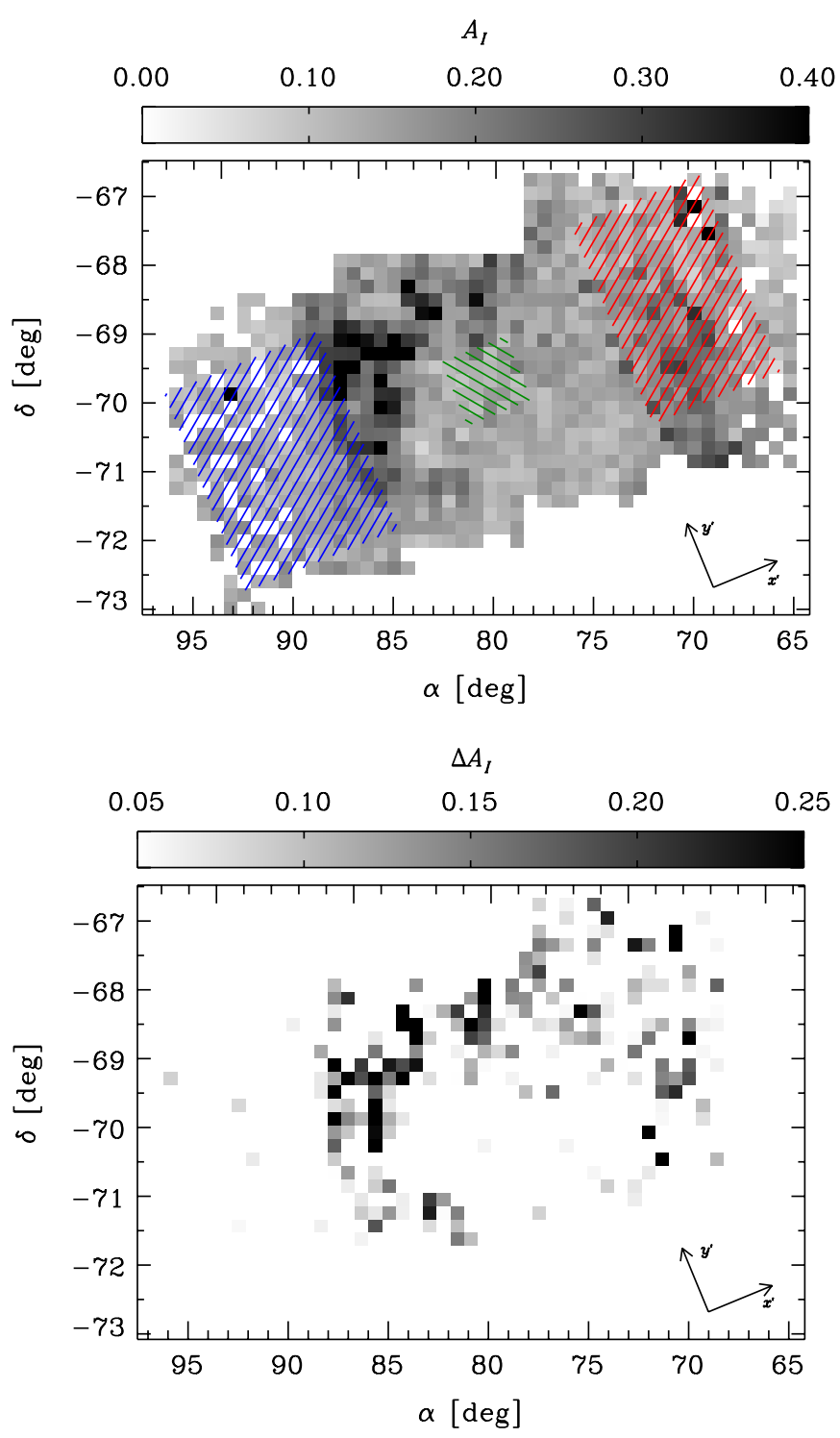

Figure 2. Upper panel: extinction map of the LMC. We calculated the mean value of $A_{I}$ in pixels of size $0.2 \times 0.2$ containing at least two RRab stars. Otherwise, we assign $A_{I}=0$. The blue, green, and red regions from Figure 1 are marked here, too. Lower panel: extinction variation along the line-of-sight $\Delta A_{I}$. We plot only pixels with $\Delta A_{I} \geqslant 0.05 \mathrm{mag}$.

(A color version of this figure is available in the online journal.)

is plotted in the upper panel of Figure 2. Comparison with the extinction maps of Zaritsky et al. (2004) reveals the same basic structure. There is a high-extinction $\left(A_{I} \gtrsim 0.4 \mathrm{mag}\right)$ region near 30 Doradus extending toward north-west and a north-south filament of increased extinction $\left(A_{I} \sim 0.25 \mathrm{mag}\right)$ at $\alpha \sim 70^{\circ}$. Our map also shows the low extinction central region seen in the Zaritsky et al. (2004) maps.

Equipped with an estimated distance $z$ to every star, we can estimate the line-of-sight structure of the extinction. To this end, we select pixels with at least four stars and split the stars into two equal groups based on their line-of-sight distance. We calculate the mean $A_{I}$ of each group and then subtract these two values to obtain the difference in extinction $\Delta A_{I}$ between distant and close stars. The values of $\Delta A_{I}$ are positive if the more distant stars have higher extinction. The distribution of values of $\Delta A_{I}$ is skewed to positive values with robust standard deviation of $0.063 \mathrm{mag}$ and skewness of 1.48 . 


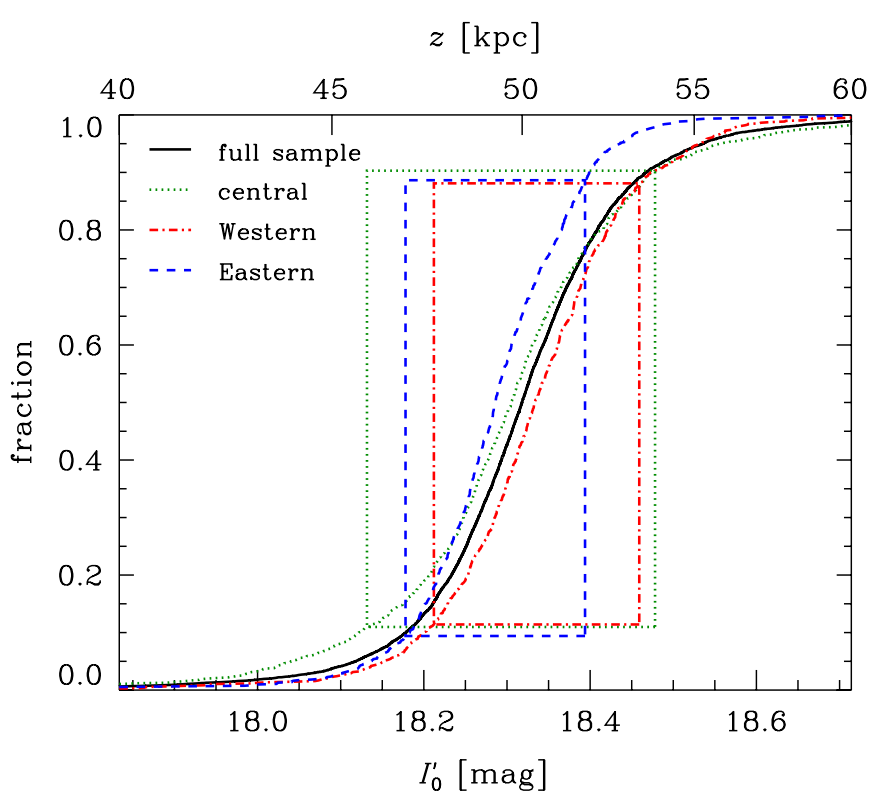

Figure 3. Cumulative distributions of extinction-corrected RRab magnitudes $I_{0}^{\prime}$ for all 9393 stars (black solid line) and those in the regions marked in Figure 1. The eastern region is shown with dashed blue line, central region with dotted green line and western region with dash-dotted red line). The boxes near the center of the plot denote areas of $68 \%$ confidence areas around the mean.

(A color version of this figure is available in the online journal.)

We plot $\Delta A_{I}$ as a function of position on the sky in the lower panel of Figure 2. As expected, the high-extinction regions seen in the upper panel of Figure 2 show up also in the internal reddening plot in the lower panel. A similar map of internal reddening was constructed by Subramanian \& Subramaniam (2009) from the spread of colors of red clump stars measured by the Magellanic Cloud Photometric Survey. Their map also shows high internal reddening close to 30 Doradus, but the north-south high-extinction filament at $\alpha \sim 70^{\circ}$ is not clearly visible.

\subsection{Structure of the Stellar Halo}

In order to determine scatter of our distance estimation method we first select two regions (the blue and red areas in Figure 1) containing about 900 stars and lying near the edges of the covered area where we expect the intrinsic depth to be lowest. We can then attribute the measured width of the distance distribution to the internal scatter of our distance estimation method. Cumulative distributions of $I_{0}^{\prime}$ for the selected regions are shown in Figure 3 with dashed blue and dash-dotted red lines, respectively. The widths of the distributions of $I_{0}^{\prime}$ measured by the standard deviations are $0.108 \mathrm{mag}(2.5 \mathrm{kpc}$ at $z=50 \mathrm{kpc})$ and $0.123 \mathrm{mag}(2.9 \mathrm{kpc})$ for the eastern and western regions, respectively, and are shown in Figure 3 with blue dashed and red dash-dotted rectangles. We assume that the scatter of our distance estimation method is that of the Eastern region, i.e., $0.108 \mathrm{mag}$ or $2.5 \mathrm{kpc}$. We note that the scatter of periodluminosity and period-color relations gives $0.08 \mathrm{mag}(1.9 \mathrm{kpc})$ as the scatter of our distance estimation method. Hence, our estimate of scatter in the distances is conservative.

We expect the depth of the LMC to be highest close to the center. We plot in Figure 3 cumulative distribution (green dotted line) of $I_{0}^{\prime}$ of about 1100 stars in a $1^{\circ} \times 1^{\circ}$ region centered on $\left(\alpha_{0}, \delta_{0}\right)$. The cumulative distribution is significantly wider than that of the side regions and also of the full sample that is shown
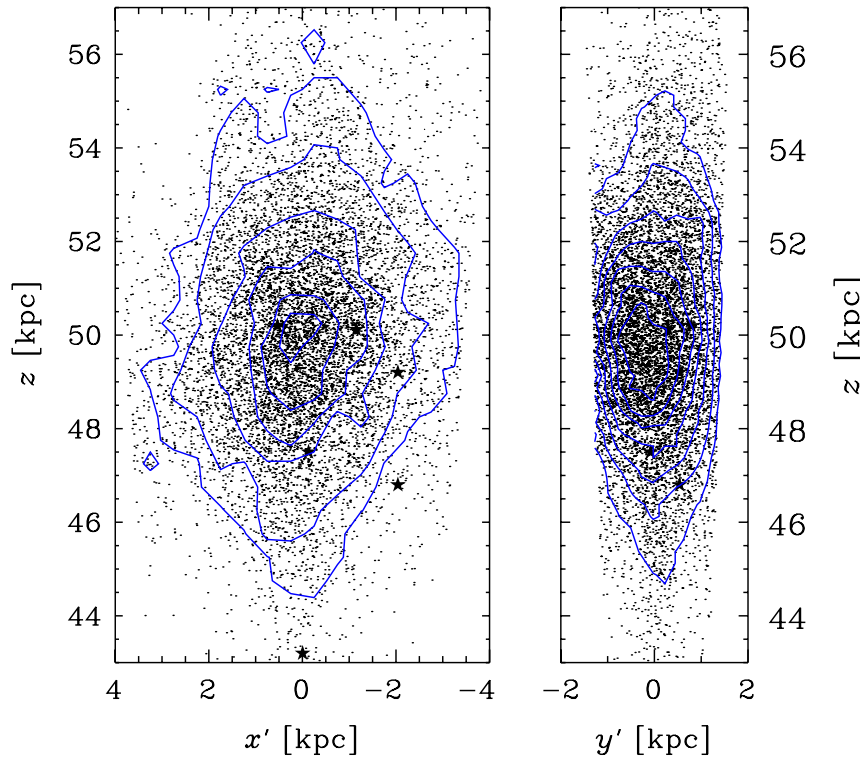

Figure 4. Line-of-sight distance $z$ of stars in our final sample as a function of rotated coordinates $x^{\prime}$ and $y^{\prime}$. Blue lines mark contours of density $50,100,200,300,400$, and $500 \mathrm{kpc}^{-2}$ for the $\left(x^{\prime}, z\right)$ plane and $100,200,300,400,500,600$, and $700 \mathrm{kpc}^{-2}$ for the $\left(y^{\prime}, z\right)$ plane. Black stars mark the positions of the eclipsing binaries shown in Figure 1; note that we plot two distances for HV 2274 (Groenewegen \& Salaris 2001; Ribas et al. 2000) and that HV 5936 falls outside the limits of the right panel.

(A color version of this figure is available in the online journal.)

with black line. The standard deviation of this subsample is $0.173 \mathrm{mag}(4.1 \mathrm{kpc})$ and is shown in Figure 3 with a green dotted rectangle. Using the scatter of our distance estimation method determined above, we can determine the intrinsic dispersion of depth in the central region to be $0.135 \mathrm{mag}$ or $3.21 \mathrm{kpc}$ (FWHM of $0.318 \mathrm{mag}$ or $7.56 \mathrm{kpc}$ ). As the intrinsic depth in the Eastern region most likely differs from zero, we can consider this value as a lower estimate. For comparison, the standard deviations of the distribution along axes of symmetry in the plane of the sky are 1.52 and $0.89 \mathrm{kpc}$, respectively. This means that the LMC RRab population depth is roughly twice the spatial extent in the plane of the sky.

From Figure 3, we also see that the distributions for the eastern and western regions are shifted with respect to each other, in the sense that the eastern region is on average about $0.05 \mathrm{mag}$ $(1.1 \mathrm{kpc})$ closer to us than the western region. Indeed, stellar populations of the LMC are known to be a part of an disk with a line of nodes at the position angle $\theta=151.0 \pm 2.4$ (Nikolaev et al. 2004) and inclined so that the eastern side is closer to the observer. From the RRab stars, we now see that the halo is also deformed so that the eastern side is closer. Encouraged by these result, we now examine the full three-dimensional distribution of RRab stars.

The population of RRab stars in the LMC can be approximated by a triaxial ellipsoid. To determine the axis ratios and space orientation of the ellipsoid we first convert equatorial coordinates for every star to physical coordinates $(\alpha, \delta) \rightarrow(x, y)$ with the centroid of our sample corresponding to the coordinate origin, $\left(\alpha_{0}, \delta_{0}\right) \rightarrow(0,0)$, and keeping $x$ to increase to the east. We then construct the covariance matrix of the coordinates (essentially the inertia tensor) and determine its eigenvalues and eigenvectors. The ratios of the square roots of the eigenvalues are the ratios of the axes and the eigenvectors correspond to their spatial directions (for a similar analysis see e.g., Paz et al. 


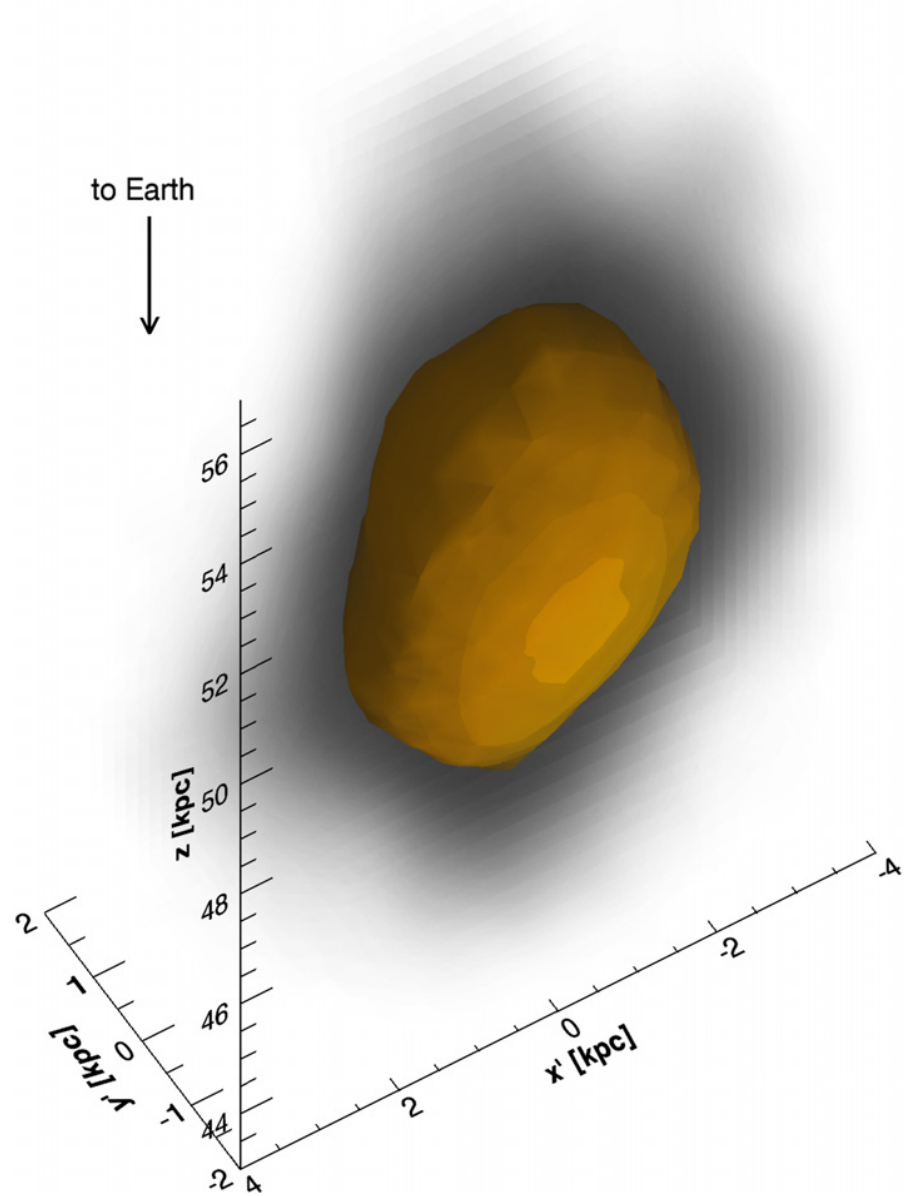

Figure 5. Three-dimensional distribution of LMC RRab stars in $\left(x^{\prime}, y^{\prime}, z\right)$ coordinates. The full distribution is displayed in semi-transparent gray and an isodensity surface of $56 \mathrm{kpc}^{-3}$ is shown in dark yellow. The visualization was constructed from $0.5 \times 0.5 \times 0.5 \mathrm{kpc}^{-3}$ cubic voxels smoothed with a boxcar width of two elements.

(A color version of this figure is available in the online journal.)

2006). We first apply this method only in the plane of the sky described by the $(x, y)$ coordinates and find that the distribution of RRab stars is elongated with an axis ratio of 1.71 and a major axis position angle of 112.4 . We use this result to define new rotated coordinates, $(x, y) \rightarrow\left(x^{\prime}, y^{\prime}\right)$. Lines parallel to the longer and shorter sides of the rectangle in Figure 1 correspond to $x^{\prime}$ and $y^{\prime}$ axes, respectively. The results for the distribution in the plane of the sky are robust as they depend only on the precisely measured positions.

Now we repeat the same procedure for the $(x, y, z)$ coordinates, however, here the line-of-sight distance $z$ is smeared by the internal scatter of our method. To correct for this, we subtract the value of internal scatter determined above from the appropriate element of the covariance matrix. We obtain the axis ratios of 1:2.00:3.50, where the longest axis is inclined by $6^{\circ}$ from the line of sight and the position angle of the projection of the ellipsoid on the plane of the sky is 113:4.

The finite sky coverage of our data can bias the determination of axis ratios and directions. To see how serious this problem is we remove stars lying outside the 250 stars per square degree contour shown in Figure 1 and repeat the procedure. We get the axis ratios of $1: 1.36: 3.53$ with the longest axis inclined by $3^{\circ}$ from the line of sight. The decrease in the relative length of the second longest axis is caused by removing relatively more stars along $x^{\prime}$ than along $y^{\prime}$.

Figure 4 shows the spatial distribution of RR Lyr stars in the $\left(x^{\prime}, z\right)$ and $\left(y^{\prime}, z\right)$ planes with overplotted density contours. Looking on the left panel we confirm our result that the Eastern part (with $x^{\prime}>0$ ) is on average closer to us than the Western part. In Figure 5 we give a more intuitive visualization of the spatial distribution of RRab stars in the LMC using a three-dimensional plot of an isodensity surface.

\section{DISCUSSION AND CONCLUSION}

In this section, we first discuss possible systematics that might be present in our analysis, then we compare our results to previous studies and conclude with implications for the structure of the LMC and future work.

\subsection{Possible Systematics}

There are a number of possible systematic errors that could lower the significance of our results. First of all, the assumption underlying all of our reasoning is that the population of RRab stars is homogeneous throughout the LMC. While we have no a priori reason to assume this, there are several checks for systematic errors. For example, comparing the period distribution of stars in our three regions, we find a mean period difference of about 0.003 days, or about 0.01 mag in the periodluminosity relation. Another check is that the distributions of light curve Fourier coefficients show no differences between these regions above the level of $3 \%$ difference in the mean. In short, we do not see any evidence of different RR Lyr populations across the LMC.

In our extinction correction of Equation (2), we set $R_{V I}$ to a value lower than the fiducial $R_{V I}=1.55$. We repeated our analysis with several values in the range $1.00 \leqslant R_{V I} \leqslant 1.55$ and we found that the distance distribution widths tend to increase with increasing $\left|R_{V I}-1.10\right|$. For example, for $R_{V I}=1.55$ we found standard deviations of $0.118,0.118$ and 0.191 mag for our eastern, western, and central regions, respectively. Furthermore, for values of $R_{V I} \gtrsim 1.2$ we find inconsistencies, in the sense that stars closer to us have a higher average extinction than the more distant stars. Or in other words, the mean $\Delta A_{I}$ was found to be about -0.016 mag compared to $0.028 \mathrm{mag}$ for $R_{V I}=1.10$. Lower values of ratio of total to selective extinction to the LMC were found also by Misselt et al. (1999).

The cuts we applied on period, amplitude and magnitude tend to reduce the outliers and consequently the derived dispersion in depth. For example, applying no cuts yields standard deviation of $0.35 \mathrm{mag}$ for the central region and $0.20 \mathrm{mag}$ for the side regions; also the central region is on average significantly brighter than the side regions for all reasonable values of $R_{V I}$. Making our cuts even more stringent does not yield significantly smaller dispersions (or reduce the difference between the central and side regions). From this analysis we conclude that our cuts efficiently remove contaminated stars and that contamination of our sample is insignificant.

We did not apply any cuts on color in order not to bias the derived shape in case there were large-scale extinction variations. However, applying an additional color cut of $0.3 \leqslant$ $(V-I) \leqslant 0.9$ removes highly reddened stars, but does not change our results. Specifically, the intrinsic line-of-sight standard deviation in the central region would be 0.123 mag $(2.91 \mathrm{kpc})$ and the axis ratio would be 1:1.99:3.14 with the 
longest axis inclined by $9^{\circ}$ from the line of sight. The application of the color cut changes our results on the intrinsic depth and axis ratios and directions only slightly.

\subsection{Comparison with Other Work}

Minniti et al. (2003) and Borissova et al. (2004, 2006) obtained radial velocities of 137 RR Lyr stars and found a very high velocity dispersion of about $50 \mathrm{~km} \mathrm{~s}^{-1}$ suggesting that the stars form a kinematically hot stellar halo. Our results on the depth dispersion are comparable with the results of Clementini et al. (2003), who analyzed RR Lyr stars in two regions close to the bar and found a depth dispersion between $3.3 \mathrm{kpc}$ and $3.8 \mathrm{kpc}$, especially if we realize that the estimate of intrinsic scatter of our method is based on outer parts of the halo which probably have substantial depth too. Subramaniam (2006) used a smaller sample of RR Lyr stars from OGLE-II (Soszyński et al. 2003) to derive the scale height of $3.0 \pm 0.9 \mathrm{kpc}$, which translates to dispersion of about $6.0 \pm 1.8 \mathrm{kpc}$. This is higher than our result, the difference stems most likely from the fact that we estimated extinction correction on a star-by-star basis and we applied stringent cuts to remove contaminations.

Nikolaev et al. (2004) found a number of Cepheids lying significantly $(>7 \mathrm{kpc})$ both in front and behind the main disk of the LMC. Similarly, the distance determinations to eclipsing binaries in the LMC show scatter larger than the uncertainties (e.g., Groenewegen \& Salaris 2001; Fitzpatrick et al. 2003; Pietrzyński et al. 2009). Both types of objects are considerably younger and more metal rich than RR Lyr stars, but it is possible that the LMC halo contains a fraction of younger objects. A population of objects in a non-spherical halo elongated along the line of sight could help to explain the number of observed microlensing events towards the LMC (Alcock et al. 2000b; Evans \& Kerins 2000; Alves 2004; Calchi Novati et al. 2006; Wyrzykowski et al. 2009). As the microlensing cross-section depends basically linearly on the lens-source distance, doubling this distance with constant halo mass yields roughly twice cross section of the self lensing due to halo of the LMC.

\subsection{Conclusion and Future Work}

We have investigated the structure of the LMC halo using fundamental mode RR Lyr stars. We found that the halo lineof-sight depth is variable, in the sense that the depth is higher in the central areas than at the edges. Furthermore, the eastern side of the halo is in general closer to us than the western side, mimicking in part the LMC disk. We were also able to provide extinction maps of the LMC and resolve the internal extinction.

While there is a significant increase of covered area between OGLE-II and OGLE-III catalogs, a survey of RR Lyr stars far from the axis of symmetry of their distribution on the sky would help to better characterize their spatial distribution. It is possible that far from the center the distribution of RR Lyr stars might exhibit an overdensity similarly to Cepheids, that are organized in a ring with an off-center bar (Nikolaev et al. 2004).
Furthermore, Borissova et al. (2006) found little variation in the velocity dispersion as a function of distance from the center of rotation, but their sample has only two stars in the farthest $[2.0 ; 2.5] \mathrm{kpc}$ bin. The OGLE-III catalogue (Soszyński et al. 2009) gives stars as far as $5 \mathrm{kpc}$ from the center and it would be interesting to see whether the difference of the line-of-sight depth between the central and side regions appears also in radial velocity measurements.

We are grateful to the OGLE team for making their data publicly available. We are grateful to C. S. Kochanek for detailed and useful comments and careful reading of the manuscript. We thank S. Dong and I. Soszyński for discussions and careful reading of the manuscript, and to the anonymous referee for suggestions.

\section{REFERENCES}

Alcock, C., et al. 2000a, AJ, 119, 2194

Alcock, C., et al. 2000b, ApJ, 542, 281

Alves, D. R. 2004, ApJ, 601, L151

Benkő, J. M., Bakos, G. Á., \& Nuspl, J. 2006, MNRAS, 372, 1657

Borissova, J., Minniti, D., Rejkuba, M., Alves, D., Cook, K. H., \& Freeman, K. C. 2004, A\&A, 423, 97

Borissova, J., Minniti, D., Rejkuba, M., \& Alves, D. 2006, A\&A, 460, 459

Cacciari, C., Corwin, T. M., \& Carney, B. W. 2005, AJ, 129, 267

Calchi Novati, S., de Luca, F., Jetzer, P., \& Scarpetta, G. 2006, A\&A, 459, 407

Clement, C. M., Xu, X., \& Muzzin, A. V. 2008, AJ, 135, 83

Clementini, G., Gratton, R., Bragaglia, A., Carretta, E., Di Fabrizio, L., \& Maio, M. 2003, AJ, 125, 1309

Cole, A. A., Tolstoy, E., Gallagher, J. S., III, \& Smecker-Hane, T. A. 2005, AJ, 129,1465

Evans, N. W., \& Kerins, E. 2000, ApJ, 529, 917

Fitzpatrick, E. L., Ribas, I., Guinan, E. F., DeWarf, L. E., Maloney, F. P., \& Massa, D. 2002, ApJ, 564, 260

Fitzpatrick, E. L., Ribas, I., Guinan, E. F., Maloney, F. P., \& Claret, A. 2003, ApJ, 587,685

Groenewegen, M. A. T., \& Salaris, M. 2001, A\&A, 366, 752

Kinman, T. D., Stryker, L. L., Hesser, J. E., Graham, J. A., Walker, A. R., Hazen, M. L., \& Nemec, J. M. 1991, PASP, 103, 1279

Lah, P., Kiss, L. L., \& Bedding, T. R. 2005, MNRAS, 359, L42

Minniti, D., Borissova, J., Rejkuba, M., Alves, D. R., Cook, K. H., \& Freeman, K. C. 2003, Science, 301, 1508

Misselt, K. A., Clayton, G. C., \& Gordon, K. D. 1999, ApJ, 515, 128

Nikolaev, S., Drake, A. J., Keller, S. C., Cook, K. H., Dalal, N., Griest, K., Welch, D. L., \& Kanbur, S. M. 2004, ApJ, 601, 260

Paz, D. J., Lambas, D. G., Padilla, N., \& Merchán, M. 2006, MNRAS, 366, 1503

Pietrzyński, G., et al. 2009, ApJ, 697, 862

Ribas, I., Fitzpatrick, E. L., Maloney, F. P., Guinan, E. F., \& Udalski, A. 2002, ApJ, 574, 771

Ribas, I., et al. 2000, ApJ, 528, 692

Schlegel, D. J., Finkbeiner, D. P., \& Davis, M. 1998, ApJ, 500, 525

Soszyński, I., et al. 2003, Acta Astron., 53, 93

Soszyński, I., et al. 2009, Acta Astron., 59, 1

Subramaniam, A. 2006, A\&A, 449, 101

Subramanian, S., \& Subramaniam, A. 2009, A\&A, 496, 399

van der Marel, R. P., Alves, D. R., Hardy, E., \& Suntzeff, N. B. 2002, AJ, 124, 2639

Wyrzykowski, Ł., et al. 2009, MNRAS, 397, 1228

Zaritsky, D., Harris, J., Thompson, I. B., \& Grebel, E. K. 2004, AJ, 128, 1606 